\title{
Correspondence
}

\section{Isomerization of Asp7 leads to increased toxic effect of amyloid- $\beta 42$ on human neuronal cells}

\author{
VA Mitkevich ${ }^{1,2}$, IY Petrushanko ${ }^{1,2}$, YE Yegorov ${ }^{1}$, OV Simonenko ${ }^{1}$, KS Vishnyakova ${ }^{1}$, AA Kulikova ${ }^{1}$, PO Tsvetkov ${ }^{1}$, AA Makarov ${ }^{* 1}$ \\ and SA Kozin ${ }^{1}$
}

Cell Death and Disease (2013) 4, e939; doi:10.1038/cddis.2013.492; published online 28 November 2013

Subject Category: Neuroscience

\section{Dear Editor,}

The generation of toxic oligomers during the aggregation of the 42-residue amyloid- $\beta(\mathrm{A} \beta)$ peptide $\mathrm{A} \beta 42$ into amyloid fibrils and plaques has emerged as a central feature of the onset and progression of Alzheimer's disease (AD). ${ }^{1}$ Isomerization of an aspartic acid residue at position 7 is a common chemical modification of $A \beta 42$ isolated from brain of the patients diagnosed with $A D .^{2}$ This modification crucially influences such processes as zinc ion chelation by $\mathrm{A} \beta,{ }^{3}$ zinc-dependent oligomerization of $\mathrm{A} \beta,{ }^{4}$ and hydrolysis of $\mathrm{A} \beta$ by the angiotensinconverting enzyme. ${ }^{5}$ As each of these molecular events is closely related to the aggregation ability of $\mathrm{A} \beta$, we have hypothesized that $A \beta 42$ containing isoaspartate 7 (iso $A \beta 42$ ) might have a role in the development of cerebral $\beta$-amyloidosis. Indeed, recently we have shown that when iso $A \beta 42$ is administered into blood of transgenic mice used as an animal model of $A D$, it accelerates the formation of amyloid plaques in contrast to $\mathrm{A} \beta 42$, which does not have this effect. ${ }^{6}$ Thus, one could rationally suggest that iso $\mathrm{A} \beta 42$ acts as aggregation seed and/or corruptive template compelling the physiological pool of endogenous $\mathrm{A} \beta 42$ to be converted into oligomers and consequently into aggregates. To get a deeper insight into the mechanism of iso $A \beta 42$ pathogenicity, in the present study a comparison was made of the cytotoxic effect of iso $A \beta 42$ and $A \beta 42$.

Human neural stem cells NSC-hTERT that underwent differentiation have been used. $^{7}$ Both isoA $\beta 42$ and $\mathrm{A} \beta 42$ peptides induce the NSC-hTERT cell death and destruction of the neural network formed by them (Supplementary Figure S1a), however toxic effects of isoA $\beta 42$ are manifested to a greater extent (Supplementary Figure S1b). Percentage of the dying cells, relative to control in the population in the presence of 10 and $15 \mu \mathrm{M}$ of isoA $\beta 42$ increased by $37 \%$ and $61 \%$, respectively, whereas the increase in the presence of $A \beta 42$ was by $11 \%$ and $32 \%$, respectively. The difference in the mechanism of cytotoxic activity of $A \beta 42$ and iso $A \beta 42$ is that the effect of iso $A \beta 42$ is by three quarters caused by the induction of apoptosis, and only one-fourth of the cells dies via necrosis path, whereas in the case of $A \beta 42$ the number of cells dying by apoptosis and necrosis is the same (Supplementary Figure S1b). Thus, the iso $A \beta 42$ toxic action on neuronal cells is not only more effective but also more specific than that of $A \beta 42$. Apoptotic effect of both peptides is associated with reduced mitochondrial potential of cells (Supplementary Figure S1c), which indicates the start of the mitochondrial apoptosis pathway. ${ }^{8}$ The action of peptides $\mathrm{A} \beta 42$ and iso $\mathrm{A} \beta 42$ leads to a decrease in intracellular glutathione level (Supplementary Figure S1c). Depletion of intracellular glutathione is also one of the causes of cell death.

In summary, it has been established for the first time that isomerization of the Asp7 residue leads to a significant increase of cytotoxic properties of $A \beta 42$. Neurotoxic effect of iso $A \beta 42$ is due to the induction of apoptosis and not damage of the cytoplasmic membrane. According to our data, the mechanism of the pathological development of $A D$ may include direct neurotoxic effect of iso $A \beta 42$ on human cells, thus, iso $A \beta 42$ appears to be a promising drug target in the therapy of $A D$ pathology.

\section{Conflict of Interest}

The authors declare no conflict of interest.

Acknowledgements. This work was supported by the Molecular and Cellular Biology Program and by the Program 'Fundamental sciences to medicine' of the Russian Academy of Sciences, by the Russian Federal Program (contract 14.512.11.0017) and by the Russian Foundation for Basic Research (13-04-40108-comfi).

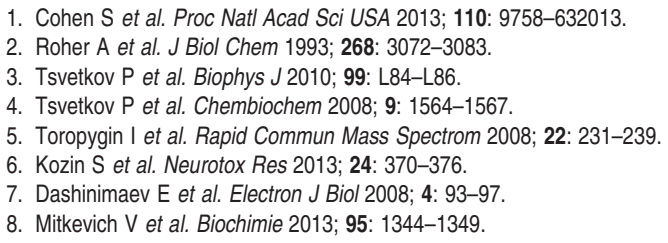

Cell Death and Disease is an open-access journal published by Nature Publishing Group. This work is licensed under a Creative Commons Attribution-NonCommercialShareAlike 3.0 Unported License. To view a copy of this license, visit http://creativecommons.org/licenses/by-nc-sa/3.0/

\section{Supplementary Information accompanies this paper on Cell Death and Disease website (http://www.nature.com/cddis)}

\footnotetext{
${ }^{1}$ Engelhardt Institute of Molecular Biology, Russian Academy of Sciences, Vavilov Street 32, Moscow 119991, Russia

*Corresponding author: AA Makarov, Laboratory of Conformational Stability of Proteins and Physical Methods of Analysis, Engelhardt Institute of Molecular Biology, Russian Academy of Sciences, Vavilov Street 32, Moscow 119991, Russia. Tel: +7 499 1359824; Fax: +7 495 1351405; E-mail: aamakarov@ eimb.ru

${ }^{2}$ These authors contributed equally to this work.
} 